\title{
Quasiballistic phonon transport from first principles
}

\author{
Pol Torres,,${ }^{1,2}$ Miquel Royo, ${ }^{1}$ Miquel López-Suárez,,${ }^{1,3}$ Junichiro Shiomi, ${ }^{2}$ and Riccardo Rurali $\odot^{1, *}$ \\ ${ }^{1}$ Institut de Ciència de Materials de Barcelona, ICMAB-CSIC, Campus UAB, 08193 Bellaterra, Spain \\ ${ }^{2}$ Department of Mechanical Engineering, The University of Tokyo, 7-3-1 Hongo, Bunkyo, Tokyo 113-8656, Japan \\ ${ }^{3}$ Dipartimento di Fisica, Università di Cagliari, Cittadella Universitaria, I-09042 Monserrato (Ca), Italy
}

(Received 14 July 2020; revised 21 September 2020; accepted 12 October 2020; published 26 October 2020)

\begin{abstract}
At short length scales phonon transport is ballistic: the thermal resistance of semiconductors and insulators is quantized and length independent. At long length scales, on the other hand, transport is diffusive and resistance arises as a result of the scattering processes experienced by phonons. In many cases of interest, however, these two transport regimes coexist. Here we propose a first-principles approach to treat quasiballistic phonon transport where diffusive and ballistic phonons receive separate theoretical treatments. Partitioning the overall phonon population for a given transport length is performed examining the mean free paths obtained from the solution of the Boltzmann transport equation and allowing only diffusive phonons to participate in anharmonic phononphonon scattering processes. We present results for $\mathrm{Si}$ and diamond, discussing the crossover from ballistic to diffusive transport as the length scale and/or the temperature increases and compute the relative contribution of ballistic and diffusive phonons to the thermal conductance in each transport condition.
\end{abstract}

DOI: 10.1103/PhysRevB.102.144305

\section{INTRODUCTION}

Heat in insulators is carried by phonons and the value of the thermal conductivity stems from the scattering processes that they experience. Phonons are scattered by impurities, boundaries and as a result of anharmonic processes, i.e., phonon-phonon scattering. In the diffusive regime the thermal resistance scales linearly with the length of the specimen $L$ because when phonons travel longer lengths they suffer more scattering events. Conversely, if the characteristic length is shorter than the mean free path (MFP), i.e., the average distance traveled by phonons between collisions, the transport is ballistic: the thermal resistance is not determined by scattering, is quantized and length independent. Both these transport regimes are described by wellconsolidated theoretical frameworks that allow predictively computing the thermal conductivity/conductance from first principles [1-7].

Phonon MFPs, however, typically span a broad length scale $[8,9]$ and can go from a few nanometers up to a several micrometers, e.g., up to $20 \mu \mathrm{m}$ in $\mathrm{Si}[9,10]$ or $1 \mathrm{~mm}$ in graphene [11], just to name a few examples. Additionally, even within a given material, phonons exhibit a broad distribution of MFPs with low-frequency phonons having typically (much) longer MFPs. Therefore, in many situations of practical interest the heat transport regime is quasiballistic [12-19] where the propagation of some phonons can be described diffusively and is characterized by multiple scattering events, whereas others travel ballistically across the device.

The diffusive-to-ballistic crossover was previously studied by Jeong et al. [20] in Si within Landauer formalism and using parametric phonon-scattering rates, whereas Hua and Min-

*rurali@icmab.es nich [21] provided an analytical solution of the Boltzmann transport equation (BTE) under relaxation time approximation (RTA) for thin film transport. Vermeersch et al. [22] computed the crossplane thin-film conductivities for a variety of semiconductors at room temperature using first-principles phonon dispersions and scattering rates via a simple summation over phonon modes; a closer comparison with these results is provided below.

In this paper we discuss how to calculate the thermal conductivity/conductance from first principles for an arbitrary length of the device and, thus, a varying ballistic-diffusive phonon ratio. Our method is based on partitioning the overall phonon population into diffusive and ballistic phonons, based on the MFP distribution and calculating separately their contribution to the thermal conductivity.

\section{COMPUTATIONAL METHODS}

\section{A. Thermal conductivity from first-principles}

We focus on bulk $\mathrm{Si}$ and diamond as case studies. We calculate the second and third order interatomic force constants (IFCs) within density-functional theory as implemented in the VASP code [23] with the local density approximation for the exchange-correlation energy functional and a planewave cutoff of $246 \mathrm{eV}$ for $\mathrm{Si}$ and $400 \mathrm{eV}$ for diamond with the projector augmented-wave method [24,25]. The IFCs are calculated from finite differences, and the required displaced supercell configurations are generated with the PHONOPY [4] and THIRDORDER.PY [2] codes. We have used a $5 \times 5 \times 5$ and a $4 \times 4 \times 4$ supercell of the primitive cell for the second- and third-order IFCs, respectively.

The solution in the fully diffusive regime is obtained using the first-principles IFCs to solve the linearized BTE for phonons using an iterative method with the SHENGBTE code [2]. Within this scheme, the lattice thermal conductivity 


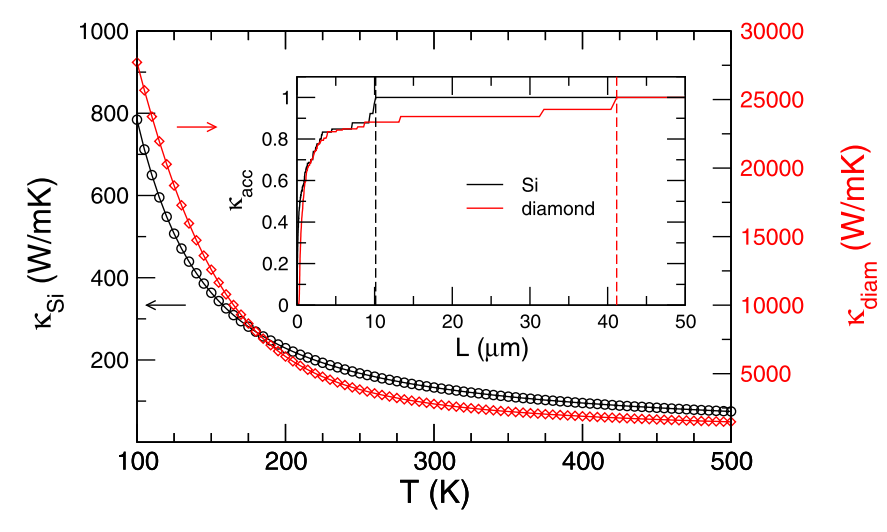

FIG. 1. Thermal conductivity as a function of temperature of $\mathrm{Si}$ and diamond without any restriction on the MFPs and without boundary scattering terms. The inset: accumulated contribution (normalized) to $\kappa$ at room temperature as a function of the phonon MFP. The dashed lines indicate the maximum MFP contributing to $\kappa$.

is obtained as

$$
\kappa^{\alpha \beta}=\frac{1}{k_{B} T^{2} V N} \sum_{\lambda} f_{0}\left(f_{0}+1\right)\left(\hbar \omega_{\lambda}\right)^{2} v_{\lambda}^{\alpha} F_{\lambda}^{\beta},
$$

where $\alpha$ and $\beta$ are the three coordinate directions $x, y$, and $z$; and $k_{B}, T, V$, and $N$ are the Boltzmann constant, the temperature, the volume of the unit cell, and the number of $\mathbf{q}$ points, respectively. The sum runs over all the phonon modes $\lambda$, which have wave-vector $\mathbf{q}$ and branch $v . f_{0}$ is the equilibrium Bose-Einstein distribution function, $\hbar$ is the reduced Planck constant, and $\omega_{\lambda}$ and $v_{\lambda}^{\alpha}$ are the phonon frequency and phonon group velocity, respectively. $F_{\lambda}^{\beta}$ is initially taken to be equal to $\tau_{\lambda} v_{\lambda}^{\beta}$, where $\tau_{\lambda}$ is the lifetime of the phonon mode $\lambda$ within the RTA. Starting from this initial guess, the solution is then obtained iteratively, and it takes the general form [2]

$$
F_{\lambda}^{\beta}=\tau_{\lambda}\left(v_{\lambda}^{\beta}+\Delta_{\lambda}^{\beta}\right)
$$

where

$$
\begin{aligned}
& \Delta_{\lambda}= \frac{1}{N} \sum_{\lambda^{\prime} \lambda^{\prime \prime}} \Gamma_{\lambda \lambda^{\prime} \lambda^{\prime \prime}}^{+}\left(\frac{\omega_{\lambda^{\prime \prime}}}{\omega_{\lambda}} \mathbf{F}_{\lambda^{\prime \prime}}-\frac{\omega_{\lambda^{\prime}}}{\omega_{\lambda}} \mathbf{F}_{\lambda^{\prime}}\right) \\
&+\frac{1}{N} \sum_{\lambda^{\prime} \lambda^{\prime \prime}} \frac{1}{2} \Gamma_{\lambda \lambda^{\prime} \lambda^{\prime \prime}}^{-}\left(\frac{\omega_{\lambda^{\prime \prime}}}{\omega_{\lambda}} \mathbf{F}_{\lambda^{\prime \prime}}+\frac{\omega_{\lambda^{\prime}}}{\omega_{\lambda}} \mathbf{F}_{\lambda^{\prime}}\right) \\
&+\frac{1}{N} \sum_{\lambda^{\prime}} \Gamma_{\lambda \lambda^{\prime}} \frac{\omega_{\lambda^{\prime}}}{\omega_{\lambda}} \mathbf{F}_{\lambda^{\prime}}, \\
& \frac{1}{\tau_{\lambda}^{0}}=\frac{1}{N} \sum_{\lambda^{\prime} \lambda^{\prime \prime}} \Gamma_{\lambda \lambda^{\prime} \lambda^{\prime \prime}}^{+}+\frac{1}{N} \sum_{\lambda^{\prime} \lambda^{\prime \prime}} \frac{1}{2} \Gamma_{\lambda \lambda^{\prime} \lambda^{\prime \prime}}^{-}+\frac{1}{N} \sum_{\lambda^{\prime}} \Gamma_{\lambda \lambda^{\prime}}
\end{aligned}
$$

In Eqs. (3) and (4), $\Gamma_{\lambda \lambda^{\prime} \lambda^{\prime \prime}}^{+}$and $\Gamma_{\lambda \lambda^{\prime} \lambda^{\prime \prime}}^{-}$are the three-phonon scattering rates, whereas $\Gamma_{\lambda \lambda^{\prime}}$ is the scattering rate from isotopic disorder [26], which in the present case is taken to be the one corresponding to the natural abundance of $\mathrm{Si}$ and $\mathrm{C}$ isotopes. This is the standard approach to calculate the thermal conductivity from first principles within an iterative approach and has been followed in several studies [27-35]. Our results are shown in Fig. 1.

\section{B. Partitioning the phonon population}

In order to address the case of a finite characteristic size $L$, we proceed as follows. At first we obtain the solution of the BTE in the RTA approximation, which is the first step of the iterative procedure described above. After that, we have access to the phonon resolved contribution to the thermal conductivity, and for each phonon we know the frequency, the velocity, and the MFP, which in the framework of the iterative solution of the BTE is defined as

$$
\Lambda_{\lambda}=\mathbf{F}_{\lambda} \frac{\mathbf{v}_{\lambda}}{\left|\mathbf{v}_{\lambda}\right|}
$$

The phonons with MFP $>L$ are unlikely to participate in any anharmonic processes and are, thus, ballistic. Therefore, when we compute the scattering rates in the following step of the iteration process, we impose the following restrictions on three-phonon processes:

$$
\begin{gathered}
\Gamma_{\lambda \lambda^{\prime} \lambda^{\prime \prime}}^{+}=0, \quad \text { if } \Lambda_{\lambda}, \Lambda_{\lambda}^{\prime}>L, \\
\Gamma_{\lambda \lambda^{\prime} \lambda^{\prime \prime}}^{-}=0, \quad \text { if } \Lambda_{\lambda}>L
\end{gathered}
$$

besides the requirement of momentum conservation $\mathbf{q}^{\prime \prime}=$ $\mathbf{q}^{\prime} \pm \mathbf{q}+\mathbf{Q}$, where $\mathbf{Q}$ is a vector of the reciprocal lattice, that must always be satisfied; $\Lambda_{\lambda}$ is the MFP of phonon $\lambda$ as defined in Eq. (5). In this way we forbid absorption processes of the kind $\lambda+\lambda^{\prime} \rightarrow \lambda^{\prime \prime}$, where, at least, one between the $\lambda$ and the $\lambda^{\prime}$ phonons is ballistic, and emission processes of the kind $\lambda \rightarrow \lambda^{\prime}+\lambda^{\prime \prime}$ when $\lambda$ is ballistic. Note, however, that processes that produce ballistic phonons are permitted, i.e., absorptions can yield phonons with $\Lambda_{\lambda^{\prime \prime}}>L$, and emissions can yield phonons with $\Lambda_{\lambda^{\prime}}$ and/or $\Lambda_{\lambda^{\prime \prime}}>L$. These phonons will be ruled out as ballistic at the subsequent step, but the momentum redistribution is permitted. The iterative process continues until a converged solution of the BTE is achieved and, at each step $n$, the conditions on $\Gamma_{\lambda \lambda^{\prime} \lambda^{\prime \prime}}^{+}$and $\Gamma_{\lambda \lambda^{\prime} \lambda^{\prime \prime}}^{-}$are enforced, based on $\left(\Lambda_{\lambda}, \Lambda_{\lambda^{\prime}}, \Lambda_{\lambda^{\prime \prime}}\right)$, i.e., the values of the MFPs, computed at step $n-1$. It should be noted that
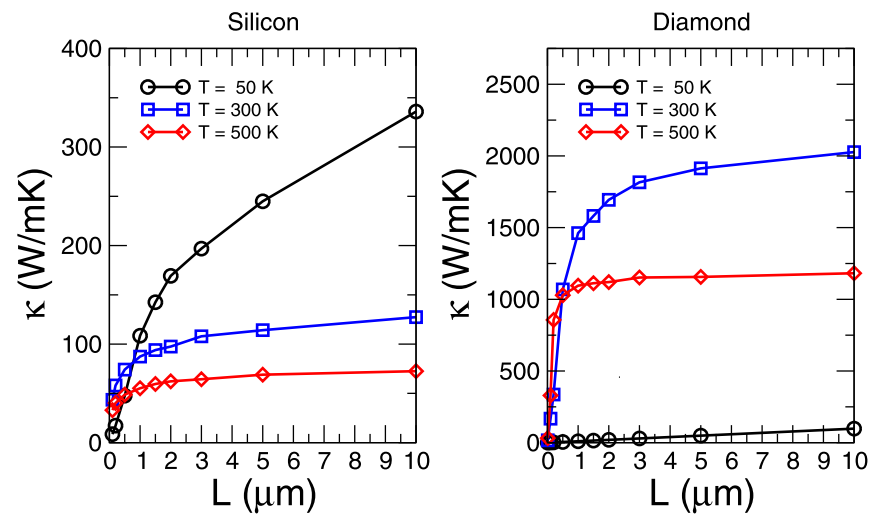

FIG. 2. Thermal conductivity as a function of the transport length at 50,300 , and $500 \mathrm{~K}$ for $\mathrm{Si}$ (left) and diamond (right). The thermal conductivity of $\mathrm{Si}$ at $50 \mathrm{~K}$ converges to the bulk value of $2385 \mathrm{~W} \mathrm{~m}^{-1} \mathrm{~K}^{-1}$ at $L \sim 560 \mu \mathrm{m}$, whereas at 300 and $500 \mathrm{~K}$ convergence is essentially achieved in the size range shown. For comparison, the experimental values of the thermal conductivity of $\mathrm{Si}$ at 50,300 , and $500 \mathrm{~K}$ are 2400,145 , and $81 \mathrm{~W} \mathrm{~m}^{-1} \mathrm{~K}^{-1}$ (see Refs. [45,46]). 
Silicon

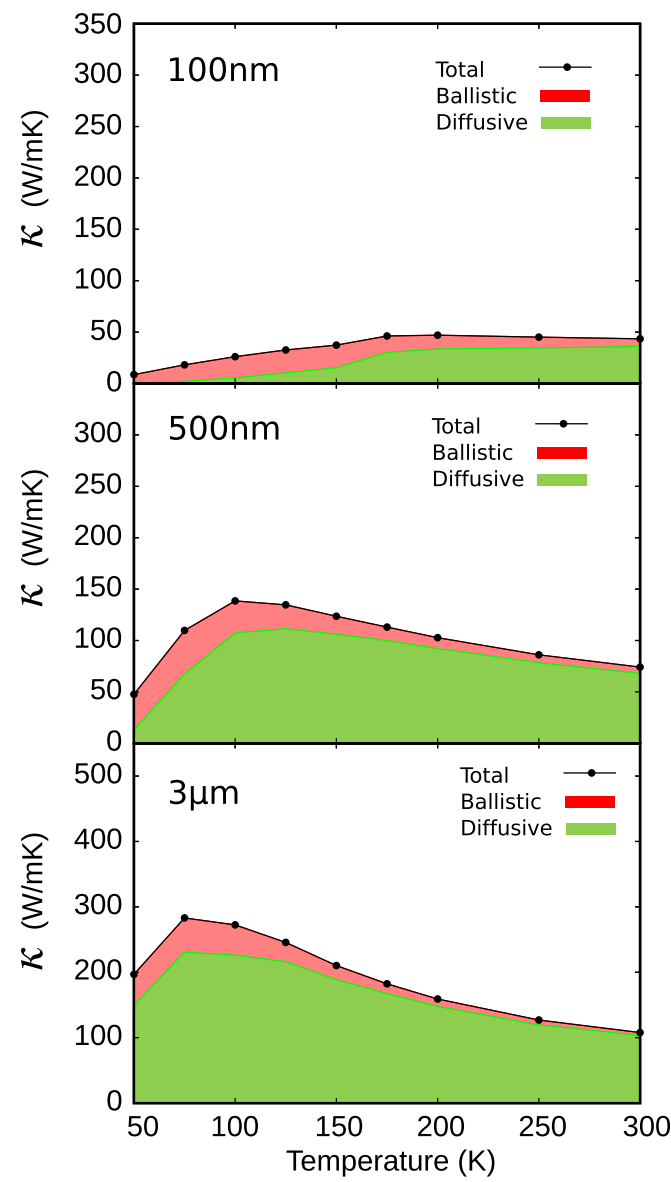

Diamond

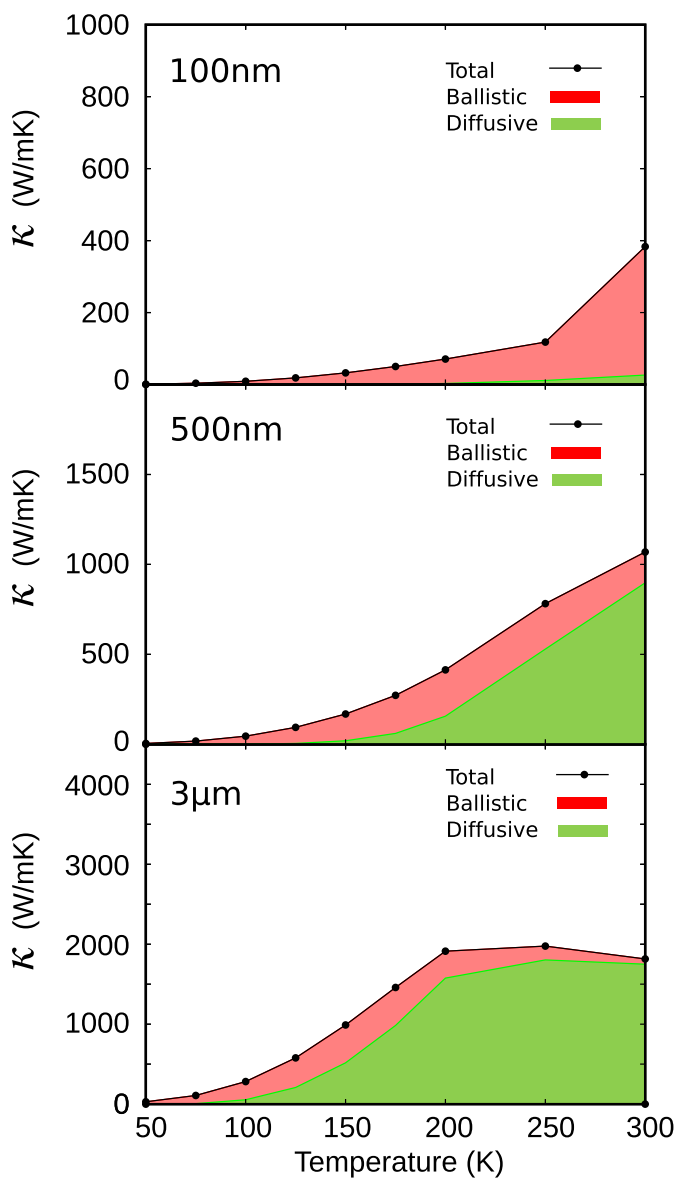

FIG. 3. Diffusive and ballistic contribution to the thermal conductivity as a function of temperature for $L=100,500 \mathrm{~nm}$, and $3 \mu \mathrm{m}$ for Si (left) and diamond (right).

within the iterative solution the concept of relaxation time should be handled with care and only $\mathbf{F}$, which can be seen as a generalized, vectorial mean free displacement, is well defined [2]. It is through $\mathbf{F}_{\lambda}$, then, that we define a scalar MFP as in Eq. (5) to be used in the formalism described above. These MFP, just like the relaxation times, can occasionally even be negative as their sign depend on the deviation of the distribution function from its equilibrium value for a mode $\lambda$. Yet, low-frequency modes that carry most of the heat are exempt from this pathological behavior (see Li et al. [2] for an exhaustive discussion) and the approximation used here is sound.

When the iterative solution of the BTE for a given $L$ is reached we know: (i) which phonons contribute to the diffusive thermal conductivity $\kappa_{\text {diff }}$, that we can compute from Eq. (1) by running the sum over the set of diffusive phonons [note that the $\kappa_{\text {diff }}$ is $\kappa$ of Eq. (1) with the conditions on the scattering rates of Eqs. (6) and (7), thus, is simply the solution of the restricted BTE]; (ii) which phonons have MFP $>L$ and must be treated as ballistic.

The conductance of ballistic phonons is obtained from the Landauer formula as

$$
G(T)=\frac{\hbar}{2 \pi \Omega} \int_{\omega_{\lambda} \in \omega_{\text {ball }}} \omega_{\lambda} \mathcal{T}\left(\omega_{\lambda}\right)\left(\frac{\partial f_{0}\left(\omega_{\lambda}, T\right)}{\partial T}\right) d \omega
$$

where $\Omega$ is the channel cross section and $\omega_{\text {ball }}$ is the set of frequencies of ballistic phonons. In the case of homogeneous materials, such as those discussed below, the transmission function $\mathcal{T}\left(\omega_{\lambda}\right)$ for each phonon is equal to one and, thus, simply counts the transport channels at a given frequency. In a more general case, assuming periodic boundary conditions in the directions perpendicular to the thermal flux, $\mathcal{T}\left(\omega_{\lambda}\right)=$ $\frac{\Omega}{(2 \pi)^{2}} \int \Xi\left(\omega_{\lambda}, \mathbf{q}_{\perp}\right) d \mathbf{q}_{\perp}$, where $\Xi\left(\omega_{\lambda}, \mathbf{q}_{\perp}\right)$ is the phonon transmission function calculated from first principles at a discrete point $\left(\mathbf{q}_{\perp}\right)$ of the two-dimensional transverse Brillouin zone by means of the Caroli formula [36].

In the present case, Eq. (8) can be rewritten as a summation over $\mathbf{q}$ in order to work directly with the outputs of SHENGBTE reducing the postprocessing. By considering $d \omega=v_{\|} d q_{\|}$, where $v_{\|}$and $q_{\|}$are the components of the velocity and the $\mathbf{q}$ vector along the transport direction, which here we take to be the [100] crystal axis, we obtain

$$
G(T)=\frac{1}{N V} \sum_{\omega_{\lambda} \in \omega_{\text {ball }}} \frac{1}{2 \pi k_{B} T^{2}} \hbar \omega_{\lambda} \frac{e^{\hbar \omega_{\lambda} / k_{B} T}}{\left(e^{\hbar \omega_{\lambda} / k_{B} T}-1\right)^{2}} v_{\|},
$$

where $N$ and $V$ are numbers of $\mathbf{q}$ points in the mesh sampling and the volume of the unit cell, respectively.

In the process described above the temperature plays a twofold role: (i) In the iterative solution the BTE establishes 
which phonons are ballistic as it determines their MFPs; (ii) once we know which phonons are ballistic, it populates them and yields the individual contribution to the thermal conductance through Eq. (9). It should be noted that, strictly speaking, the thermal conductivity is an ill-defined magnitude within a ballistic picture, at variance with the thermal conductance $G$, which can always be defined and computed. Yet, as is customary in the experimental literature, we will discuss an effective thermal conductivity where a ballistic conductivity $\kappa_{\text {ball }}=G L / \Omega$, that increases linearly with $L$ adds up to the diffusive conductivity $\kappa_{\text {diff }}$, which is constant.

\section{RESULTS AND DISCUSSION}

We applied this formalism to the case study of bulk Si and diamond. In Fig. 2 we display our results for the thermal conductivity as a function of length of these two materials at three representative temperatures: 50, 300, and $500 \mathrm{~K}$. As expected, the higher the temperature, the more diffusive is the phonon transport regime due to the increase in the phonon-phonon scattering rates and the subsequent decrease inthe MFPs and, thus, of the phonon-phonon scattering rates. Therefore, at the relatively low temperature of $50 \mathrm{~K} \mathrm{Si}$ exhibits nondiffusive features, whose signature is a thermal conductivity that increases with length up to a few micrometers. Conversely, at higher temperatures, a constant thermal conductivity is recovered at shorter lengths, indicating the lack of measurable effects of ballistic transport. Phonon MFPs in diamond are considerably longer than in $\mathrm{Si}$ as shown in the inset of Fig. 1. Therefore, ballistic effects are observed even at moderately high temperatures and, when $\mathrm{Si}$ and diamond are compared at the same temperature, the latter behave nondiffusively up to much longer lengths. Our approach naturally gives access to the ballistic-diffusive ratio, which is well visualized in Fig. 3 where we plot the relative contribution of both transport regimes to $\kappa(T)$ at some selected values of $L$. This is an important result because, whereas the qualitative trends were expected (ballistic transport becomes dominant at low $T$ and short $L$ ), it quantitatively accounts for such a behavior and fully maps the ballistic-diffusive ratio in the $(T, L)$ space. These plots reveal that at ultrashort lengths, $L=100 \mathrm{~nm}$, ballistic effects dominate phonon transport in diamond and are still non-negligible in $\mathrm{Si}$ up to room temperature. For $L=500 \mathrm{~nm}$ ballistic phonons propagation is still predominant up to $200 \mathrm{~K}$ for diamond, whereas diffusive transport takes over in Si already at $100 \mathrm{~K}$. In micrometer samples transport is essentially diffusive for $\mathrm{Si}$ throughout almost all the temperature range considered, whereas a significant contribution from ballistic phonons in diamond is still observable at $200-250 \mathrm{~K}$.

We now compare the solution obtained within the approach here proposed and the one of the standard phonon BTE. Noteworthy, the BTE not only accounts for the fully diffusive limit $L \rightarrow \infty$, but it also naturally yields the thermal conductance in the ballistic limit. The phonon lifetime $\tau_{\lambda}$ of Eq. (1) results from different scattering mechanisms, each one characterized by its own lifetime: $\tau_{\mathrm{ph} \text {-ph }}$ for anharmonic phonon-phonon processes, $\tau_{i}$ and $\tau_{b}$ for impurity and boundary scattering. The total $\tau_{\lambda}$ can be obtained from Matthiessen's rule as $\tau_{\lambda}^{-1}=\tau_{\mathrm{ph}-\mathrm{ph}}^{-1}+\tau_{i}^{-1}+\tau_{b}^{-1}$ as in Eq. (4), although there
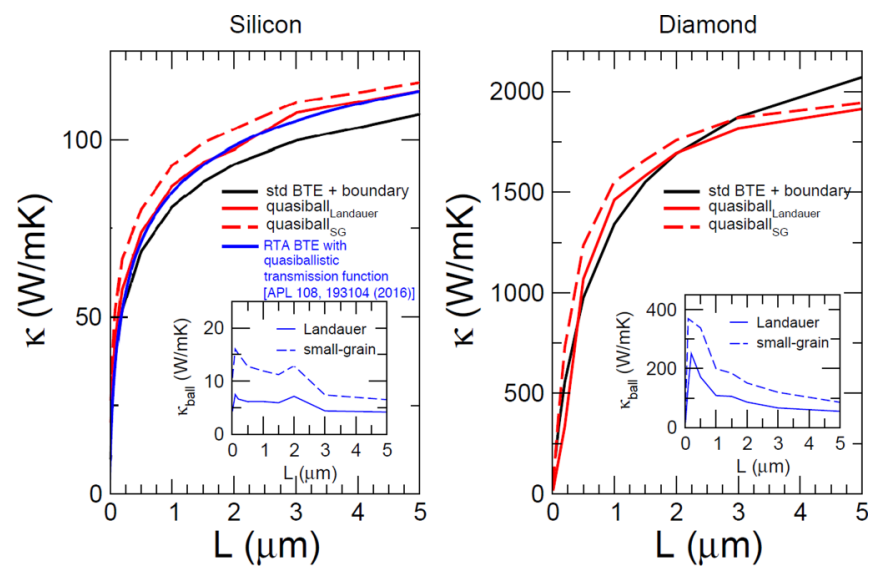

FIG. 4. Thermal conductivity as a function of length at $300 \mathrm{~K}$ for $\mathrm{Si}$ and diamond after partitioning the phonon population into diffusive and ballistic phonons (red lines) or adding an effective boundary scattering term within the BTE (black line). Continuous and dashed red lines correspond to different ways of calculating the ballistic contribution: Landauer vs small grain; a detailed comparison is shown in the insets. In the case of $\mathrm{Si}$ we also display (blue line) the computed cross-plane thin-film thermal conductivity for $\mathrm{Si}$ at room temperature using first-principles phonon dispersions and scattering rates via a simple summation over phonon modes as obtained in Ref. [22].

the contribution from $\tau_{b}$ was not explicitly accounted for. In the limit of $L \rightarrow 0, \tau_{b}$ is much smaller than $\tau_{\text {ph-ph }}$ and $\tau_{i}$ and, thus, $\tau_{\lambda} \sim \tau_{b}$. Taking the expression for the boundary lifetime, $\tau_{b}=L / \mathbf{v}$ is easy to show that one recovers the Landauer conductance from Eq. (1) (we consider here the case of specular scattering; otherwise the expression for $\tau_{b}$ can be generalized by introducing a specularity parameter [37]). Therefore, at the standard BTE level, both the ballistic and the diffusive limits are correctly reproduced. On the other hand, the diffusiveballistic crossover is heavily approximated: All the phonons are considered to be diffusive, but their contribution is suppressed with a suppression factor that depends on the $\tau_{b} / \tau_{\mathrm{ph} \text {-ph }}$ and $\tau_{b} / \tau_{i}$ ratios. To estimate the extent of this approximation and compare it with our solution, we have considered a boundary scattering term in the unrestricted solution of the BTE. To this end, we have added a boundary lifetime $\tau_{b}$, through Matthiessen's rule to $\tau_{\lambda}$ of the RTA initial guess of the iterative solution of $F_{\lambda}^{\beta}$. In Fig. 4 we compare these results with those obtained with our approach at $T=300 \mathrm{~K}$. As can be seen, the introduction of an effective boundary scattering term leads to a considerable underestimation of the thermal conductivity in the quasiballistic regime with respect to our results where phonon population is partitioned into diffusive and ballistic phonons. The difference in the value of $\kappa$ is larger than $30 \%$ at $L=100 \mathrm{~nm}$ for Si and $L=500 \mathrm{~nm}$ for diamond and then tends to vanish in the fully diffusive limit. Yet, for $\mathrm{Si}$ it is on the order of the $15-20 \%$ at $L=3 \mu \mathrm{m}$. Interestingly, our results agree well with those of Ref. [22], which rely on the phonon transmission function $\operatorname{Tr}=2 \Lambda / L /(1+2 \Lambda / L)$ that naturally captures a gradual and smooth crossover between fully ballistic transport $[\operatorname{Tr}(\Lambda \gg L) \rightarrow 1]$ and fully diffusive transport $[\operatorname{Tr}(\Lambda \ll L) \rightarrow 0]$ without the need to separate phonons into distinct groups. 
Silicon

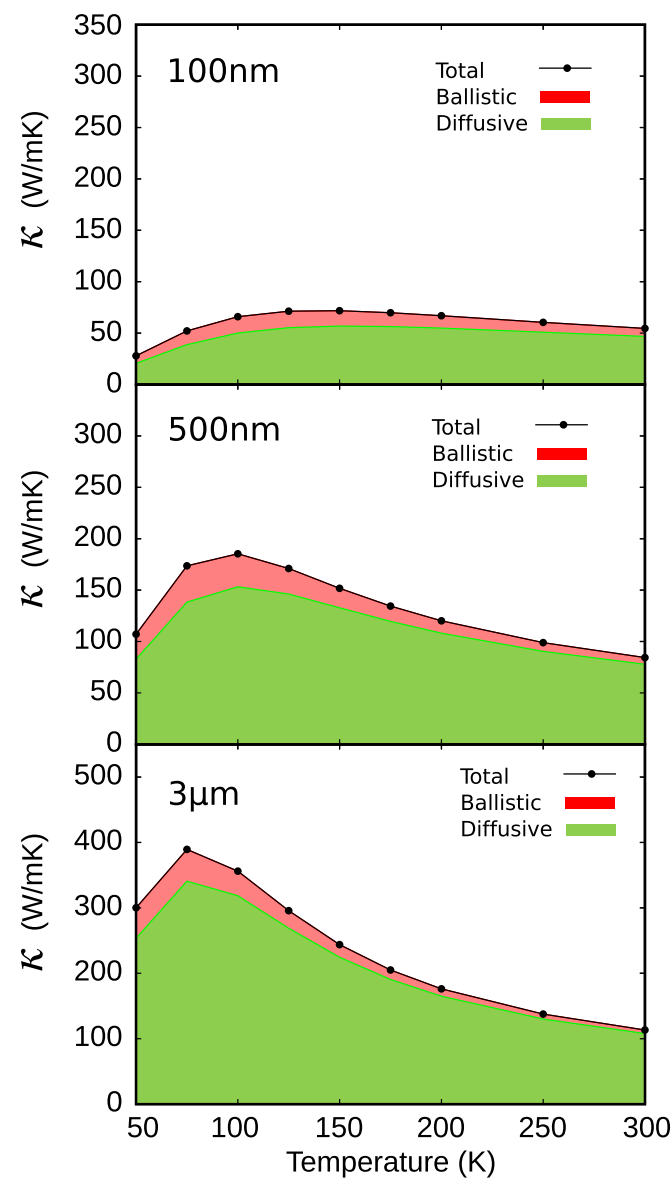

Diamond

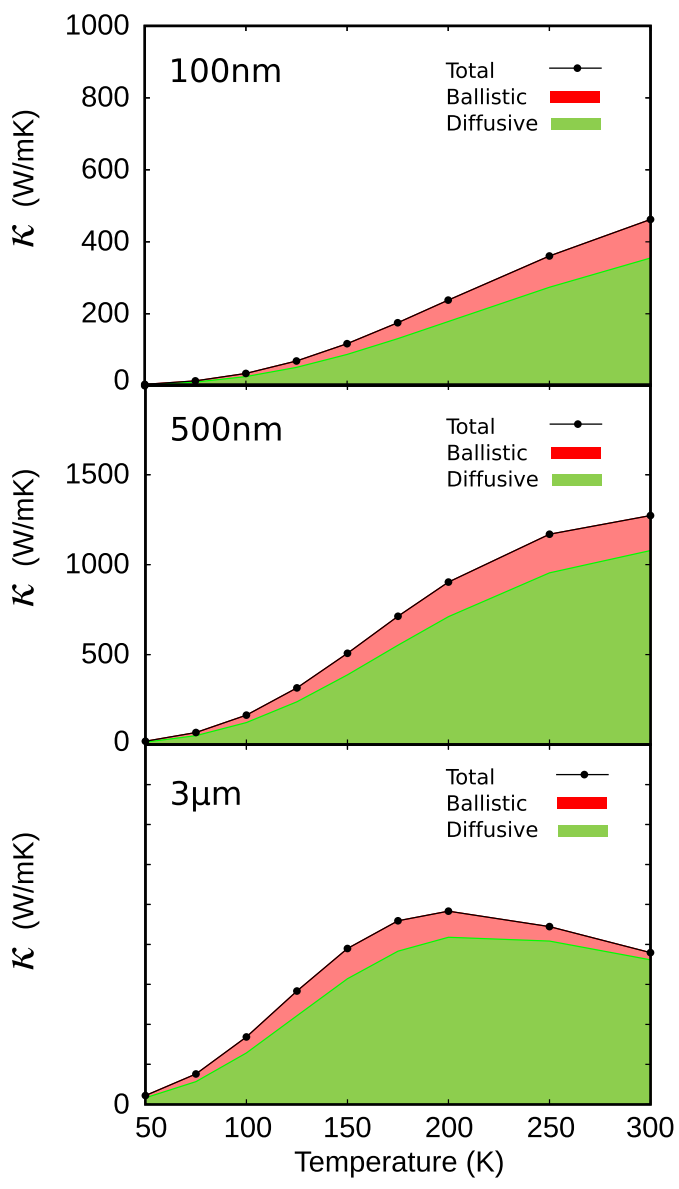

FIG. 5. Diffusive and ballistic contribution to the thermal conductivity as a function of temperature for $L=100,500 \mathrm{~nm}$, and $3 \mu \mathrm{m}$ for Si (left) and diamond (right) obtained as a weighted sum over phonon modes, using a weight factor of $e^{-(L / \Lambda)}$ and of $1-e^{-(L / \Lambda)}$ for ballistic and diffusive phonons, respectively (see the text).

Provided that the phonon population is properly partitioned, similar results can also be obtained from the so-called small-grain conductivity [2] where all phonon MFPs are taken to be equal to $L$, i.e., the sample size, instead of calculating them from the anharmonic scattering rates. The small-grain [38] thermal conductivity can be expressed as

$$
\kappa_{\mathrm{sg}}^{\alpha \beta}=\frac{1}{k_{B} T^{2} V N} \sum_{\lambda} f_{0}\left(f_{0}+1\right)\left(\hbar \omega_{\lambda}\right)^{2} \frac{v_{\lambda}^{\alpha} v_{\lambda}^{\beta}}{\left|\mathbf{v}_{\lambda}\right|} .
$$

Here, rather than considering all the phonons to have their MFPs limited by $L$ as in the standard small-grain approximation, we only sum the contribution of the phonons that we previously determined to be ballistic,

$$
\kappa_{\text {ball }, \mathrm{sg}}=\sum_{\lambda \in \lambda_{\text {ball }}} \kappa_{\mathrm{sg}, \lambda},
$$

where $\lambda_{\text {ball }}$ is the set of ballistic phonons. The advantage of this approach is that both $\kappa_{\text {diff }}$ and $\kappa_{\text {ball }}$ are computed on the same q-point mesh and phonons labeled as ballistic after the self-consistent solution of the BTE that enforces the condition of MFP $<L$ are readily identified without the need of interpolations or approximations. Additionally, at variance with the Landauer formalism, we do not need to fix a transport direction, and we always work with the full $\boldsymbol{\kappa}$ tensor. The results agree reasonably well, although at a closer look the ballistic contribution at the small-grain level is systematically larger than the one computed from the Landauer formula (see the inset in Fig. 4).

We now discuss briefly the limitations of the approach here proposed and outline a convenient generalization to circumvent them. In the scheme described above we have labeled phonons as diffusive, when $\Lambda<L$ and ballistic, when $\Lambda>$ $L$. However, the MFP is the average distance traveled by phonons between collisions. The travel distance can be taken to be exponentially distributed with $\Lambda$ as mean,

$$
p(\ell)=\frac{1}{\Lambda} e^{-(\ell / \Lambda)} .
$$

Therefore, the probability that a phonon undergoes a scattering event within a distance $L$ is given by

$$
P[0 \leqslant \ell \leqslant L]=\int_{0}^{L} p(\ell) d \ell=1-e^{-(L / \Lambda)} .
$$

Within this more nuanced framework, it is both true that phonons with small mean free paths $(\Lambda<L)$ have in reality a small but nonzero chance to reach the system boundary without scattering and that phonons with long mean free paths 
$(\Lambda>L)$ can experience a scattering event before crossing the device length. A more rigorous treatment would, therefore, be to label the phonons stochastically: A phonon has a probability $e^{-(L / \Lambda)}$ to be assigned to the ballistic set and a probability $1-e^{-(L / \Lambda)}$ to be assigned to the diffusive set. The main inconvenient of such a randomized labeling is that each run of the iteration scheme would result in a different conductivity, which should then be averaged over several independent trials. Another possibility to go beyond the sharp cutoff criterion to label phonons consists in obtaining the converged MFPs from the conventional iterative BTE solution, and then computing the total conductivity as a weighted sum over phonon modes of a ballistic contribution (weight factor $e^{-(L / \Lambda)}$ ) and diffusive contribution (weight factor $1-e^{-(L / \Lambda)}$ ). The results obtained with this approach and shown in Fig. 5 are qualitatively consistent with those of Fig. 3 where we adopted the cruder rule to label phonons, although we now obtained a somewhat larger fraction of diffusive phonons. Indeed, by looking at Eq. (13), one sees that for a phonon with MFP equal to the system size $(\Lambda=L)$ this probability becomes $1-e^{-1} \sim 0.63$. Therefore, for MFPs near the system size it is still more likely than not $(P>0.5)$ that a scattering event will actually take place and, thus, a larger fraction of diffusive phonons is the be expected.

\section{CONCLUSIONS}

To summarize, we have presented a simple, but rigorous approach to the calculation of thermal conductivity in the quasiballistic transport regime from first principles. Our method relies on well-consolidated theoretical frameworks and numerical tools. It is based on properly partitioning diffusive and ballistic phonons and switching off certain scattering processes. Specifically, phonons with MFPs longer than the characteristic transport size (e.g., the device length) are forbidden to take part in phonon-phonon scattering processes. Besides predicting the thermal conductivity in more realistic conditions, the approach proposed is able to quantitatively account for the relative contribution of diffusive and ballistic phonons to $\kappa$. The characteristic size of the sample $L$ is implicitly assumed to be the distance between the hot and the cold reservoirs so that the transverse dimension do not play a role in limiting the thermal conductivity. The formalism here proposed, however, can be extended to the case of one-dimensional conductors, such as nanowires [17,39-42] as long as it can be assumed that their vibrational properties can be approximated with those of the bulk and that the rupture of translational symmetry perpendicular is accounted for by means of position-dependent phonon lifetimes [2,43,44]. We have presented results for $\mathrm{Si}$ and diamond, showing that ballistic effects at room temperature can survive up to a few hundreds nanometers for $\mathrm{Si}$ and to $5 \mu \mathrm{m}$ for diamond. In diamond, ballistic phonons dominate heat transport at ultrashort lengths up to room temperature and in submicron samples up to $200 \mathrm{~K}$. Conversely, in Si the larger contribution always comes from diffusive phonons unless one goes to ultrashort lengths and very low temperatures where ballistic phonons yield a measurable contribution to the thermal conductivity.

\section{ACKNOWLEDGMENTS}

We acknowledge financial support by the Ministerio de Economía, Industria y Competitividad (MINECO) under Grant No. FEDER-MAT2017-90024-P and the Severo Ochoa Centres of Excellence Program under Grant No. SEV-20150496 and by the Generalitat de Catalunya under Grant No. 2017 SGR 1506. M.L.-S. was funded through a Juan de la Cierva fellowship. We thank the Centro de Supercomputación de Galicia (CESGA) for the use of their computational resources. P.T. acknowledges funding by the Canon Foundation in Europe. The authors thank M. Brandbyge and L. Colombo for critical reading of the paper and J. Carrete for useful discussions about technical details related with the implementation of SHENGBTE.
[1] K. Esfarjani and H. T. Stokes, Phys. Rev. B 77, 144112 (2008).

[2] W. Li, J. Carrete, N. A. Katcho, and N. Mingo, Comp. Phys. Commun. 185, 1747 (2014).

[3] A. Togo, L. Chaput, and I. Tanaka, Phys. Rev. B 91, 094306 (2015).

[4] A. Togo and I. Tanaka, Scr. Mater. 108, 1 (2015).

[5] G. Fugallo and L. Colombo, Phys. Scr. 93, 043002 (2018).

[6] L. G. C. Rego and G. Kirczenow, Phys. Rev. Lett. 81, 232 (1998).

[7] W. Zhang, T. S. Fisher, and N. Mingo, Numer. Heat Trans. B Fundam. 51, 333 (2007).

[8] A. J. Minnich, J. A. Johnson, A. J. Schmidt, K. Esfarjani, M. S. Dresselhaus, K. A. Nelson, and G. Chen, Phys. Rev. Lett. 107, 095901 (2011).

[9] T. Feng and X. Ruan, J. Nanomater. 2014, 206370 (2014).

[10] P. Torres, A. Torelló, J. Bafaluy, J. Camacho, X. Cartoixà, and F. X. Alvarez, Phys. Rev. B 95, 165407 (2017).

[11] G. Fugallo, A. Cepellotti, L. Paulatto, M. Lazzeri, N. Marzari, and F. Mauri, Nano Lett. 14, 6109 (2014).
[12] M. E. Siemens, Q. Li, R. Yang, K. A. Nelson, E. H. Anderson, M. M. Murnane, and H. C. Kapteyn, Nature Mater. 9, 26 (2010).

[13] A. J. Minnich, Phys. Rev. Lett. 109, 205901 (2012).

[14] T. Yamamoto, S. Konabe, J. Shiomi, and S. Maruyama, Appl. Phys. Express 2, 095003 (2009).

[15] M.-H. Bae, Z. Li, Z. Aksamija, P. N. Martin, F. Xiong, Z.-Y. Ong, I. Knezevic, and E. Pop, Nat. Commun. 4, 1734 (2013).

[16] D. Bagchi and P. K. Mohanty, J. Stat. Mech.: Theory Exp. (2014) P11025.

[17] R. Anufriev, S. Gluchko, S. Volz, and M. Nomura, ACS Nano 12, 11928 (2018).

[18] A. Sood, F. Xiong, S. Chen, R. Cheaito, F. Lian, M. Asheghi, Y. Cui, D. Donadio, K. E. Goodson, and E. Pop, Nano Lett. 19, 2434 (2019).

[19] A. E. Sachat, F. Köenemann, F. Menges, E. D. Corro, J. A. Garrido, C. M. S. Torres, F. Alzina, and B. Gotsmann, 2D Mater. 6, 025034 (2019).

[20] C. Jeong, S. Datta, and M. Lundstrom, J. Appl. Phys. 111, 093708 (2012).

[21] C. Hua and A. J. Minnich, J. Appl. Phys. 117, 175306 (2015). 
[22] B. Vermeersch, J. Carrete, and N. Mingo, Appl. Phys. Lett. 108, 193104 (2016).

[23] G. Kresse and J. Furthmüller, Phys. Rev. B 54, 11169 (1996).

[24] P. E. Blöchl, Phys. Rev. B 50, 17953 (1994).

[25] G. Kresse and D. Joubert, Phys. Rev. B 59, 1758 (1999).

[26] S.-i. Tamura, Phys. Rev. B 27, 858 (1983).

[27] F. Zhou, W. Nielson, Y. Xia, and V. Ozoliņš, Phys. Rev. Lett. 113, 185501 (2014).

[28] L. Zhu, G. Zhang, and B. Li, Phys. Rev. B 90, 214302 (2014).

[29] J. Carrete, N. Mingo, and S. Curtarolo, Appl. Phys. Lett. 105, 101907 (2014).

[30] C. W. Li, J. Hong, A. F. May, D. Bansal, S. Chi, T. Hong, G. Ehlers, and O. Delaire, Nat. Phys. 11, 1063 (2015).

[31] J. He, M. Amsler, Y. Xia, S. S. Naghavi, V. I. Hegde, S. Hao, S. Goedecker, V. Ozolinšs, and C. Wolverton, Phys. Rev. Lett. 117, 046602 (2016).

[32] M. Zeraati, S. M. Vaez Allaei, I. Abdolhosseini Sarsari, M. Pourfath, and D. Donadio, Phys. Rev. B 93, 085424 (2016).

[33] A. Katre, J. Carrete, B. Dongre, G. K. H. Madsen, and N. Mingo, Phys. Rev. Lett. 119, 075902 (2017).

[34] J. A. Seijas-Bellido, H. Aramberri, J. Íñiguez, and R. Rurali, Phys. Rev. B 97, 184306 (2018).

[35] P. Torres, J. Íñiguez, and R. Rurali, Phys. Rev. Lett. 123, 185901 (2019).

[36] C. Caroli, R. Combescot, P. Nozieres, and D. Saint-James, J. Phys. C: Solid State Phys. 4, 916 (1971).
[37] J. M. Ziman, Electrons and Phonons (Oxford University Press, Oxford, 1960), p. 463.

[38] We keep the nomenclature of small-grain for consistency with Ref. [2], where it was introduced. To avoid misunderstandings, however, we stress that the grain in this context is nothing more than the sample size. We are not attempting at describing multicrystalline samples or grain boundaries with their associated thermal boundary resistance.

[39] R. Rurali, Rev. Mod. Phys. 82, 427 (2010).

[40] T.-K. Hsiao, H.-K. Chang, S.-C. Liou, M.-W. Chu, S.-C. Lee, and C.-W. Chang, Nat. Nanotechnol. 8, 534 (2013).

[41] H. Zhang, C. Hua, D. Ding, and A. J. Minnich, Sci. Rep. 5, 9121 (2015).

[42] D. Vakulov, S. Gireesan, M. Y. Swinkels, R. Chavez, T. Vogelaar, P. Torres, A. Campo, M. De Luca, M. A. Verheijen, S. Koelling, L. Gagliano, J. E. M. Haverkort, F. X. Alvarez, P. A. Bobbert, I. Zardo, and E. P. A. M. Bakkers, Nano Lett. 20, 2703 (2020).

[43] W. Li, N. Mingo, L. Lindsay, D. A. Broido, D. A. Stewart, and N. A. Katcho, Phys. Rev. B 85, 195436 (2012).

[44] W. Li, L. Lindsay, D. A. Broido, D. A. Stewart, and N. Mingo, Phys. Rev. B 86, 174307 (2012).

[45] C. J. Glassbrenner and G. A. Slack, Phys. Rev. 134, A1058 (1964).

[46] A. V. Inyushkin, A. N. Taldenkov, A. M. Gibin, A. V. Gusev, and H.-J. Pohl, Phys. Status Solidi C 1, 2995 (2004). 